\title{
A Study on the Relationship Between Problematic Internet Use, Emotional Regulation Difficulties and Aggressive Behavior of Vocational High School Students
}

\author{
CongTan ${ }^{1}$, Simeng Dong ${ }^{2,}$, Qinnan Dong ${ }^{3}$ \\ ${ }^{1}$ School of Marxism Studies, Chongqing University of Posts and Telecommunications, Chongqing, China \\ ${ }^{2}$ School of Economics and Management, Chongqing University of Posts and Telecommunications, Chongqing, China \\ ${ }^{3}$ Yangtze River Economic Research Center, Chongqing Technology and Business University, Chongqing, China \\ *Corresponding author. Email: dongsm@cqupt.edu.cn
}

\begin{abstract}
This study builds a moderated mediation model based on ecosystem theory. Through the study of 534 vocational high school students in Chongqing, we investigated their Internet addiction, emotional regulation difficulties, aggressive behavior and family closeness. The results show that: (1) according to demographic statistics, $38.4 \%$ of the total number of students were diagnosed with Internet addiction. (2) After controlling for gender, age, family and personal economic status, PIU had a positive predictive effect on aggressive behavior. (3) PIU indirectly affects aggressive behavior through the mediating effect of emotion regulation difficulty. (4) The indirect effect was moderated by family closeness. These results are helpful to understand how family intimacy affects adolescents' PIU, emotional regulation difficulties and aggressive behavior tendency from the perspective of different subsystems of developmental system theory and their interactions. The prevention and intervention of adolescent PIU should not only pay attention to their psychological state, but also improve the influence of PIU on adolescents' bad behavior from the aspect of family relationship.
\end{abstract}

Keywords: Vocational high school students, Problematic Internet use, Difficulty in emotional regulation, Aggressive behavior, Family closeness.

\section{INTRODUCTION}

Adolescent is an important period of adolescent development. The imbalance of physical and mental development will make middle school students face various psychological crises and lead to a series of psycho-behavioral problems. As of June 2020, China's youth Internet users had reached 140 million. As the Internet penetration rate increases year by year, problematic Internet use (PIU) attracts more and more attention of researchers ${ }^{[1]-[2]}$.

Previous studies have found that the degree of Internet addiction is significantly positively correlated with emotional regulation difficulties ${ }^{[3]}$. Teenagers are in the stage of strong curiosity and strong thirst for knowledge, they are often more easily addicted to the Internet cannot extricate themselves, especially some unknown source of false information and violent content of information often make them suffer, so that they are more likely to show aggressive behavior. However, the tendency of aggressive behavior not only causes physical and mental harm to adolescents and others ${ }^{[4]-[5]}$, but also has the tendency of aggressive behavior in some adolescents who have criminal records ${ }^{[6]}$.

According to Developmental Contextualism and Bioecological Systems theory ${ }^{[7]}$, individual development is the result of continuous interaction between individuals and the surrounding environment. Adolescence is an important transition period from dependence on family to relative independence, and the influence of family is the main proximal factor leading to adolescent substance use and crime ${ }^{[8]-[9]}$.

Therefore, we believe that it is necessary to have a more in-depth discussion on the use of PIU among adolescents in vocational high schools, the risks and mechanisms of PIU, which is conducive to scientific prevention, effective control and correct guidance of PIU 
among adolescents. The purpose of this study was to investigate the effect of PIU on aggressive behavior of vocational high school students, and to investigate the mechanism of emotional regulation difficulties and family closeness.

\section{LITERATURE REVIEW AND RESEARCH HYPOTHESIS}

Problematic Internet use (PIU), also known as Internet addiction, excessive Internet use, and pathological Internet use ${ }^{[10]}$. An aressive behavior refers to any kind of behavior that purposefully or intentionally harms or attempts to harm the physical or psychological condition of another individual or to destroy other objects. Adolescents with Internet addiction have stronger tendency of verbal aggression, physical aggression and self-venting [11]-[12]. Therefore, we hypothesize that adolescent PIU increases their aggressiveness.

H1: Adolescent PIU has an obvious positive correlation with their aggressive behavior.

As one of the important variables of family environment, the relationship between family closeness and PIU has been highly valued by researchers ${ }^{[13]}$. Two of the core functions of a good parent-child relationship (emotional warmth and social control) help reduce PIU in adolescents. The probability of Internet addiction among adolescents in parents' family environment with marital conflict is significantly increased [14]-[15]. Therefore, the following assumptions are proposed:

$\mathrm{H} 2$ : Family closeness plays a regulatory role in the relationship between PIU and emotional regulation difficulties.

H3: Family closeness plays a moderating role in the relationship between PIU and aggressive behavior.

H4: Family closeness plays a moderating role in the relationship between emotional regulation difficulties and aggressive behavior.

Emotional regulation is a dynamic process of monitoring and regulating internal emotional processes and external behaviors to adapt to the needs of external situations and interpersonal relationships [16]-[17]. Emotional regulation difficulties are significantly correlated with individual interpersonal relationship, social interaction, happiness, etc. ${ }^{[18]}$. Good emotion regulation is of great significance to individual development. Based on this, we propose the following assumptions, and the research framework is shown in Figure 1.

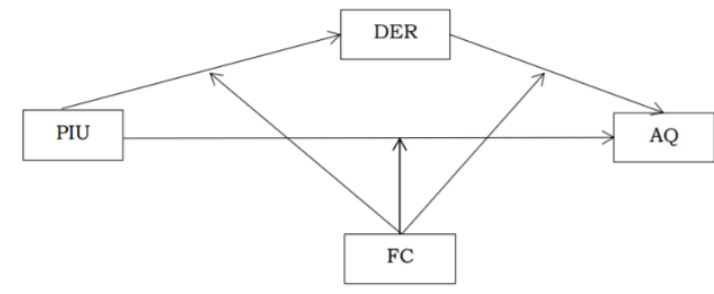

Figure 1. Research framework

Note: $\mathrm{PIU}=$ Problematic Internet use, $\mathrm{DER}=$ Difficulty in emotional regulation, $\mathrm{AQ}=$ Aggressive behavior, $\mathrm{FC}=$ Family closeness.

H5: Difficulty in emotional regulation plays a mediating role between problematic Internet use and aggressive behavior in adolescents.

\section{PROCEDURE}

\subsection{Data source}

This study selected a vocational high school in Chongqing, a total of 612 students to complete the questionnaire survey. After eliminating 80 invalid questionnaires (irregular answers), 532 valid questionnaires were finally obtained, with an effective rate of $86.9 \%$. All participants received written informed consent, and participants received no rewards. The subjects included 260 boys and 272 girls; $40.8 \%$ of the students use the Internet for entertainment (games), and the number of students who use the Internet for 4-5 hours is the most, which is 192 , accounting for $36.1 \%$ of the total number.

\subsection{Variables}

\subsubsection{Problematic Internet use scale}

The Chinese version of the Internet Addiction Test for Chinese (Young, 1998) was adopted ${ }^{[19]}$. There are 20 questions in this questionnaire, and each question is graded from 1 (almost none) to 5 (always) on a 5-point scale. A total score of 50 or less is considered as a normal Internet user, while a total score of 50 or more is considered as an Internet addict.

\subsubsection{Emotional regulation difficulty Scale}

The Emotional regulation Difficulty Scale (DERS) is derived from the research on the emotional regulation difficulties of adolescents, and has good applicability in adolescents ${ }^{[20]}$. DERS is a 36-item measure of the overall level of mood disorders. A 5-point score is adopted, from "never" to "always", and the higher the score, the more difficult the emotional regulation is. 


\subsubsection{Aggressive Behavior Questionnaire}

The Chinese version of Buss-Warren Aggression Questionnaire Revised in China (BWAQ-RC) was used to evaluate the aggressiveness tendency of the teenagers in the study and was used as the grouping basis. There were 34 items in the questionnaire, and each item was graded from 1 (not like at all) to 5 (almost the same). The evaluation results were expressed by the total score of each dimension item. High score indicated strong aggression tendency, and vice versa.

\subsubsection{Family closeness scale}

Cohesion and Cohesion scales were measured according to a simplified questionnaire [21], i.e., to measure emotional connections between family members. The questionnaire consists of nine items with a 5-point score of 1-5 points from "never" to "always". Higher scores indicate greater family closeness.

\subsubsection{Family closeness scale}

Cohesion and Cohesion scales were measured according to a simplified questionnaire [21], i.e., to measure emotional connections between family members. The questionnaire consists of nine items with a 5-point score of 1-5 points from "never" to "always". Higher scores indicate greater family closeness.

\subsection{Methods}

In this study, SPSS 24.0 was used for statistical analysis, and THE PROCESS macro (Model 4) of SPSS was used to study the mediating role of emotional regulation difficulties in Internet addiction and aggressive behavior ${ }^{[22]}$. The moderating effect of family closeness on the direct and indirect relationship between Internet addiction and aggressive behavior was studied using process macro SPSS (Model 59).

\section{RESULT}

Table 1 shows the data characteristics and Pearson correlation coefficients between the major variables. Internet addiction was positively correlated with emotional regulation difficulties and aggressive behavior, but negatively correlated with family intimacy. Emotional regulation difficulty was positively correlated with aggressive behavior and negatively correlated with family intimacy. Aggressive behavior is negatively correlated with family closeness.

Table 1. Descriptive statistics and Pearson correlation coefficient

\begin{tabular}{llllll}
\hline & Mean & SD & 1 & 2 & 3 \\
\hline 1.PIU & 2.783 & 0.977 & 1.000 & & \\
$2 . D E R$ & 2.763 & 1.004 & $.699 * *$ & 1.000 & \\
\hline
\end{tabular}

\begin{tabular}{llllll}
\hline 3.AQ & 2.698 & 0.953 & $.801 * *$ & $.732 * *$ & 1.000 \\
4.FC & 3.480 & 1.253 & - & - & - \\
& & & $.774 * *$ & $.745^{* *}$ & $.816^{* *}$ \\
\hline
\end{tabular}

Note: **Pearson correlation coefficient was significant at 0.01 level (double tail).

$\mathrm{H} 1$ and $\mathrm{H} 5$ respectively hypothesized the relationship between Internet addiction and aggressive behavior and the mediating effect of emotional regulation difficulty. The results are shown in Table 2. Internet addiction had a significant positive effect on aggressive behavior $(\beta=0.801, P<0.001)$. In addition, Internet addiction also had a significant positive effect on emotional regulation difficulties $(\beta=0.699, \mathrm{P}<0.001)$. In addition, emotional regulation had a significant regulating effect between Internet addiction and aggressive behavior (indirect effect $=0.235$, 95\% BootCIs Total $=[0.169,0.308]$, indirect effect proportion $=41.52 \%$ ). Therefore, the hypothesis $\mathrm{H} 1$ and H5 have been confirmed.

Table 2. Test of the mediating effect of emotion regulation

\begin{tabular}{llll}
\hline Variable & DER & AQ & AQ \\
\hline PIU & $.699 * * *$ & $.801 * * *$ & $.566 * * *$ \\
& $(.031)$ & $(.026)$ & $(.033)$ \\
DER & & & $.336 * * *$ \\
& & & $(.033)$ \\
R2 & .489 & .642 & .699 \\
$\mathrm{~F}$ & $506.907 * * *$ & $948.843 * * *$ & $615.292 * * *$ \\
\hline
\end{tabular}

Note: $* * * \mathrm{P}<0.001 ; * * \mathrm{P}\langle 0.01 ; * \mathrm{P}<0.05$.

$\mathrm{H} 2 \sim \mathrm{H} 4$ respectively hypothesized the moderating role of family intimacy in the pathways between Internet addiction and aggressive behavior (Table 3). As shown in the first regression results, Internet addiction significantly positively affected emotional regulation difficulties, and this relationship was significantly reduced by family closeness. Therefore, the indirect pathway between Internet addiction and emotional regulation difficulties was alleviated by family closeness $(\beta=-0.228, \quad \mathrm{SE}=0.043, \mathrm{P}<0.001)$. The relationship between Internet addiction and aggressive behavior was weaker among students with higher perceived family closeness $(\beta=0.121, \mathrm{SE}=0.046,95 \% \mathrm{CIs}=[0.030$, $0.213]$ ), while the relationship between Internet addiction and aggressive behavior was stronger among students with lower perceived family closeness $(\beta=0.063, \mathrm{SE}=$ $0.046,95 \% \mathrm{CIs}=[0.030,0.213]) . \mathrm{SE}=0.063,95 \% \mathrm{CIs}=$ $[0.534,0.782])$.

Table 3. Test of the moderating effect of family closeness

\begin{tabular}{lll}
\hline Variable & DER & AQ \\
\hline PIU & $.314 * * *$ & $.390 * * *$ \\
& .043 & $(.037)$ \\
DER & & $.123 * *$ \\
& & $(.036)$ \\
\hline
\end{tabular}




\begin{tabular}{lll}
\hline $\mathrm{FC}$ & $-.367 * * *$ & $-.252 * * *$ \\
& .050 & $(.041)$ \\
$\mathrm{PIU} * \mathrm{FC}$ & $-.228 * * *$ & $-.268 * * *$ \\
& .043 & $(.042)$ \\
$\mathrm{DER} * \mathrm{FC}$ & & -.021 \\
& & $(.042)$ \\
$\mathrm{R} 2$ & .613 & .780 \\
$\mathrm{~F}$ & $278.454 * * *$ & $372.412 * * *$ \\
\hline
\end{tabular}

Note: $* * * \mathrm{P}<0.001 ; * * \mathrm{P}<0.01 ; * \mathrm{P}<0.05$.

\section{DISCUSSION}

Under the framework of Developmental Contextualism and attachment theory, this study constructed a moderated mediation model based on "ecosystem theory", and explored the mechanism of adolescent problematic Internet use (PIU) on their aggressive behavior. At the same time, the adjustment relationship between family factors in each path was investigated.

The results showed that the percentage of vocational high school students with Internet addiction reached $38.4 \%$. PIU has a positive influence on aggressive behavior. PIU has a positive predictive effect on aggressive behavior, and indirectly affects aggressive behavior through the mediating effect of emotion regulation difficulty. Excessive Internet participation is not only related to individual's explicit aggressive behavior and intention, but also closely related to implicit aggression, especially teenagers' Internet participation and exposure to violent media. Internet media addicts hold self-aggressive beliefs and show more positive implicit attitudes towards aggression.

Family closeness plays a moderating role in the relationship between PIU and aggressive behavior, and between PIU and emotional regulation difficulty, respectively. It can be seen that family relationship has a strong influence on adolescents' problem Internet use and the bad behaviors it brings. According to the adult and environment model of parent-child relationship development, family is the most important microenvironment affecting teenagers, and family is also the first firewall to prevent teenagers from Internet addiction. Family risk and its cumulative effect can lead to adolescents' pathological Internet use behavior, while good parent-child communication can keep adolescents away from Internet addiction, and close relationship can make children have healthier psychological quality, stable mood and higher self-efficacy.

\section{CONCLUSION}

The purpose of this study was to explore the mechanism of problematic Internet use in vocational high school adolescents on their aggressive behavior and the moderating effect of family factors among various pathways. The results showed that the percentage of vocational high school students with Internet addiction reached 38.4\%. Problematic Network use (PIU) has a positive influence on aggressive behavior. PIU has a positive predictive effect on aggressive behavior, and indirectly affects aggressive behavior through the mediating effect of emotion regulation difficulty. In addition, family closeness plays a moderating role in the relationship between PIU and aggressive behavior, and between PIU and emotional regulation difficulties, respectively. It can be seen that family relationship has a strong influence on adolescents' problem Internet use and the bad behaviors it brings. These results are helpful to understand how family intimacy affects adolescents' PIU, emotional regulation difficulties and aggressive behavior tendency from the perspective of different subsystems of developmental system theory and their interactions. The prevention and intervention of adolescent PIU should not only pay attention to their psychological state, but also improve the influence of PIU on adolescents' bad behavior from the aspect of family relationship.

\section{AUTHORS' CONTRIBUTIONS}

Cong Tan: Writing-review \& editing. Simeng Dong: Data curation, Writing-review \& editing. Qinnan Dong: Conceptualization, Data curation, Formal analysis, Writing-original draft, Conceptualization, Methodology, Supervision.

\section{ACKNOWLEDGMENTS}

We would like to thank all the students who took part in the survey.

\section{REFERENCES}

[1] Spada, Marcantonio M. An overview of problematic Internet use[J]. Addictive Behaviors, 2014, 39(1):36. DOI: 10.1016/j.addbeh.2013.09.007

[2] Aboujaoude E. Problematic Internet use: an overview $[\mathrm{J}]$. World Psychiatry Official Journal of the World Psychiatric Association, 2010, 9(2):8590. DOI: 10.1002/j.2051-5545.2010.tb00278.x

[3] Chen Li-Hua. The relationship between adult attachment and Internet addiction behavior of college students[D]. Shanghai Normal University, 2020. DOI: $10.27312 /$ d.cnki.gshsu.2020.000846

[4] Li Xiao-Fang, Bian Chen-Yang, Chen Yan-Lin, et al. The characteristics of adolescent empathy development and its relationship with aggressive behavior [J]. Journal of Chinese Mental Health , 2015, 29(9): 708-713. DOI : 10.3969/j.issn.10006729.2015 .09 .013 
[5] Smokowski P R, Guo S, Evans C, et al. Risk and Protective Factors Across Multiple Microsystems Associated With Internalizing Symptoms and Aggressive Behavior in Rural Adolescents: Modeling Longitudinal Trajectories From the Rural Adaptation Project[J]. American Journal of Orthopsychiatry, 2016. DOI : 10.1037/ort0000163

[6] MD Cohn, Veltman D J, Pape L E, et al. Incentive Processing in Persistent Disruptive Behavior and Psychopathic Traits: A Functional Magnetic Resonance Imaging Study in Adolescents[J]. Biological Psychiatry, 2015:615-624. DOI : 10.1016/j.biopsych.2014.08.017

[7] Bronfenbrenner U, Ceci S J. Nature-nurture reconceptualized in developmental perspective: a bioecological model.[J]. Psychological Review, 1994, 101(4):568-586. DOI : 10.1037//0033295X.101.4.568

[8] Allen J. Autonomy and relatedness in family interactions as predictors of expressions of negative adolescent affect. Special Issue: affective processes in adolescence[J]. J Res Adolesc, 1994, 4. DOI: 10.1207/s15327795jra0404-6

[9] Nash S G, Mcqueen A, Bray J H. Pathways to adolescent alcohol use: family environment, peer influence, and parental expectations.[J]. Journal of Adolescent Health Official Publication of the Society for Adolescent Medicine, 2005, 37(1):1928. DOI : 10.1016/j.jadohealth.2004.06.004

[10] Thornberry T P, MD Krohn. Peers, drug use, and delinquency.[J]. Handbook of Antisocial Behavior, 1997:218-233. DOI https://www.mendeley.com/catalogue/6e32ccf906fe-3dec-ac3e-b903e0c48293/

[11] Lian B, Abiero B, Kamara P. Guns in the Home and the Possibility of Aggressive Tendencies: Exploring a Community Sample of Adolescents in LowIncome Communities[J]. Social Work in Public Health, 2017, 32(4):1-9. DOI : 10.1080/19371918.2017.1289873

[12] Mp A, Sv B, Ec B, et al. Problematic Internet use (PIU), personality profiles and emotion dysregulation in a cohort of young adults: trajectories from risky behaviors to addiction ScienceDirect[J]. Psychiatry Research, 2020, 289:113036. DOI:10.1016/j.psychres.2020.113036

[13] Jafar B K, Touraj H N, Jalil B K. THE RELATIONSHIP OF PSYCHOLOGICAL CAPITAL WITH SOCIAL CAPITALAMONG STUDENTS OF TABRIZ UNIVERSITY[J]. 2012.
[14] Gao T, Meng X, Qin Z, et al. Association between parental marital conflict and Internet addiction: A moderated mediation analysis[J]. Journal of Affective Disorders, 2018, 240:27-32. DOI : 10.1016/j.jad.2018.07.005

[15] Nagase S, Morita N, Ogai Y, et al. Evaluation of the relationship between internet addiction and interpersonal relationships in university studentsin Japan[J]. Nihon Arukōru Yakubutsu Igakkai zasshi=Japanese journal of alcohol studies \& drug dependence, 2016, 51 (3) :184-202.

[16] Bushman, Brad, Baumeister J, et al. Do people aggress to improve their mood? Catharsis beliefs, affect regulation opportunity, and aggressive responding. $[\mathrm{J}]$. Journal of Personality and Social Psychology, 2001, 81 ( 1 ) :17-32. DOI : 10.1037/0022-3514.81.1.17

[17] Kim, L, Gratz. Risk Factors for and Functions of Deliberate Self-Harm: An Empirical and Conceptual Review[J]. Clinical Psychology: Science and Practice, 2006. DOI 10.1093/clipsy.bpg022

[18] Liu Qi-Gang. Adolescent Emotion Regulation: Structure, Influencing Factors and Significance to School Adaptation[D]. Changchun: Jilin University, 2009.

\section{DOI : CNKI:CDMD:1.2009.098192}

[19] Wu Xian-Hua. Research on the characteristics of interpersonal relationship among adolescents with Internet addiction and its comprehensive intervention[D]. Huazhong University of Science and Technology, 2013. DOI : 10.7666/d.D409601

[20] Gratz K L, Roemer L. The relationship between emotion dysregulation and deliberate self-harm among female undergraduate students at an urban commuter university.[J]. Cognitive Behaviour Therapy, 2008, 37 ( 1 ) :14-25. DOI : $10.1080 / 16506070701819524$

[21] Fei Li-Peng, Shen Qi-Jie, Zheng Yan-Ping, et al. Preliminary evaluation of "Family Intimacy and Adaptability Scale" and "Family Environment Scale" - a comparative study of normal families and family members with schizophrenia[J]. Chinese Mental Health Journ. DOI CNKI:SUN:ZXWS.0.1991-05-001

[22] Bolin J H. Hayes, Andrew F. (2013). Introduction to Mediation, Moderation, and Conditional Process Analysis: A Regression-Based Approach. New York, NY: The Guilford Press $\lceil\mathrm{J}\rceil$. Journal of Educational Measurement, 2014, 51(3). DOI : 10.1111/jedm. 12050 